\title{
Preface to the special issue on developments of the concepts of randomness, statistics and probability
}

\author{
GIUSEPPE LONGO ${ }^{\dagger}$ and MIOARA MUGUR-SCH ÄCHTER \\ ${ }^{\dagger}$ Informatique, CNRS - Ecole Normale Supérieure et CREA, Paris, France \\ Email: Giuseppe.Longo@ens.fr \\ ${ }^{\ddagger}$ CeSEF (Centre pour la Synthèse d'une Epistémologie Formalisee) and \\ adMCR (association pour le développement de la Méthode de Conceptualisation Relativisée), \\ 47 Boulevard Georges Seurat, 92200 Neuilly-sur-Seine, France \\ Email: mms@noos.fr
}

Received 26 June 2012

Under a variety of names, and in a more or less explicit form, the concept that we now call 'probability' must have taken shape in the mind of human beings since the dawn of thought, as a nuance added to the idea of chance (randomness) or unpredictability, though chance may not be exactly the right word. Some time later, the concepts of what we now describe as 'statistics' and 'statistically stable', moved away from the idea of 'chance' and came closer to something else, which was called 'probability' and has been fuzzily conceived as being, in some sense, abstract and 'ideal'. Throughout history it has been felt that unpredictability can have degrees, and that it can be measured using probabilities.

For a very long time the idea connected with the word 'probability' has preserved this vague conceptual status as an unrealised potentiality relating to the concepts of fundamental unpredictability (chance) and partial predictability (what we now call 'statistical'), moving without a definite path along the dimensions vaguely sketched out by these two concepts within the constant flow of ever changing ideas.

It was only in the $17^{\text {th }}$ century that this idea began to acquire some structure of its own, first through the work of Blaise Pascal (1654), but mainly through Jacob Bernoulli's well-known concept of the 'law' of large numbers (which was developed from 1690 and published in 1715). Later Richard von Mises (1883-1953) further clarified the very peculiar relation within this 'law' between the recently postulated real number giving the probability of an event happening from amongst a given collection of possible events, and the sequence of rational numbers that express the evolving relative frequencies of the outcomes of this event when the associated random experiment is repeated: in the view of both Bernoulli and von Mises, these relative frequencies determine (but in an ideal noneffective sense) the numerical value of the probability of the event under consideration. Finally, in 1933, Kolmogorov gave us a genuine mathematical syntax for discussing the concept of probability. Using the general mathematical theory of measure, he fully worked out a specific syntactic entity, which he called a probability space, which is based on $a$ 
universe of elementary events and which is used to define an algebra of events, on which a probability 'measure' is postulated to 'exist': thus, everything becomes both very subtle and completely defined from a formal point of view. However, this mathematical syntax was conceived in terms of Set Theory to insure maximal generality, but in this way introduces minimal logical and semantic specifications.

This gave rise to a paradigmatic case of the problem of determining the optimality, for a given pragmatic aim, of the relations between semantics and syntax, and it is still far from being solved.

However, this problem was ignored, and continues to be ignored, and thus Kolmogorov's probabilistic syntax has been considered capable of quite satisfactorily hosting and formally organising any specific factual probabilistic problem. Furthermore, hardly anybody has seemed to be troubled by the fact that, in any given factual probabilistic situation, in order to calculate predictions, we have to specify numerically the individual probabilities of each event involved in that particular factual situation, whereas Kolmogorov's theory of probabilities contains exclusively general constraints on a probability measure, which are quite independent of any particular probabilistic situation.

Nevertheless, it has progressively, though remarkably slowly, become clear due to the pressure of the effectiveness requirements arising from the theory of computation, that the factual concept of probability inherited from Bernoulli and von Mises, and incorporated in Kolmogorov's formalisation, is not an effective concept as it stands, so it has become ever more disturbing that even now there is no general effective method for defining the distribution of the numerical probabilities of the events involved in a given particular factual probabilistic situation. The strongest reaction was that of Kolmogorov himself, and during the 1980's he kept asserting that his 'theory of probabilities' should be considered purely as a chapter in the mathematical theory of measure, and devoid of factual applicability. On the other hand he did, with collaborators, develop an algorithmic representation of complexities.

At the same time, various ideas about, and applications of, a concept of randomness were developed, but, as in the case of the concept of probability, there is still no clear consensus.

Furthermore, as is well known, specific structural details of the quantum mechanical concept of probability hinder its incorporation into Kolmogorov's classical formal concept. Recently, several authors have tried to identify the source from which the quantum probabilities could be derived instead of being postulated, but without stressing the distinction between factual and formal data, which amounts to a complexified version of the questions raised by the classical concept of probability.

Given all this, it seemed useful to dedicate a special issue of Mathematical Structures in Computer Science to encourage a critical and constructive examination of the present conceptual situation in the field of randomness/statistics/probability. In order to favour the emergence of such a result, we called on workers in several domains for contributions making any use of the concepts of chance, statistics and probability. At the same time, we announced the organisation of a final debate enabling us to draw global conclusions on current general concepts of probability, the results of which are published at the end of this special issue. 
We extend our warm thanks to the contributors for their collaboration, and we hope that this volume may contain the germs of an improved and reasonably general concept of probability.

Giuseppe Longo (Editor-in-Chief of Mathematical Structures in Computer Science) Mioara Mugur-Schächter (Guest editor of this special issue of the journal). 